\title{
Cefalea como signo de alarma ¿infradiagnóstico?
}

Idrogo-Huaranga Luis Fernando ${ }^{1, \mathrm{a}}$, Mego-Ramirez Jhan Deyvi1 ${ }^{\mathrm{a}}$, Vera-Sanchez Mario Alberto,a, Soto-Cáceres Victor ${ }^{2}$

Sr. Editor:

T a cefalea constituye un motivo de consulta frecuente en los servicios de emergencia, representando entre $1-3 \%$ de las admisiones, un paciente con cefalea genera la disyuntiva entre un síntoma primario o secundario a un trastorno neurológico ${ }^{(1)}$. El objetivo primordial del médico clínico, el cual se va a enfrentar una cefalea en emergencia es identificar con precisión las cefaleas secundarias que podrían comprometer la vida del paciente ${ }^{(2)}$.

La International Classification of Headache Disorders,define a una cefalea primaria a aquella que cumple criterios de migraña, tensional o en racimos y una secundaria como una cefalea de novo que tiene un desorden subyacente como factor causal ${ }^{(1)}$.

En la anamnesis para orientar a un diagnóstico presuntivo se utiliza la historia clínica en la mayoría de los casos; para eso se debe definir el perfil temporal del dolor (su forma de inicio) y así determinar si es súbita o no; con esto nos va a permitir orientar si se trata de una hemorragia subaracnoidea (HSA) y así poder realizar otras exploraciones complementarias así sea la cefalea de corta duración, exploración neurológica normal o si hubiera cesado la cefalea en el momento de llegar a la emergencia. ${ }^{(2,3)}$

La exploración física es un complemento de la anamnesis para poder tener un diagnóstico más preciso, aquí se debe incluir dos constantes, tanto la temperatura como la presión arterial; ya que ante la presencia de fiebre se debe de pensar en un diagnóstico como la meningitis, por otro lado los pacientes hipertensos o con presiones arteriales superiores $120 \mathrm{mmHg}$ de la diastólica y/o 200 $\mathrm{mmHg}$ de la sistólica pueden provocar cefalea. El fondo de ojo también es importante ya que la presencia de edema de papila, es un hallazgo característico de aumento en la presión intracraneal ${ }^{(4)}$

Una tomografía computarizada realizada en las seis primeras horas de síntomas súbitos de cefalea intensa, con un paciente no inconciente y sin síntomas focales suele ser suficiente para confirmar el diagnóstico de HSA siempre tenerlo en cuenta ya que, de no tomar las medidas adecuadas de manera precoz, esta enfermedad, puede desenlazar en complicaciones graves e irreversibles, incluso la muerte. ${ }^{(5)}$

Aceptado: 16/07/2020

Recibido: 16/11/2019

$1 \quad$ Universidad San Martín de Porres Filial Norte

a Estudiante de Medicina Humana

2 Docente Facultad de Medicina Universidad de San Martin de Porres Filial Norte 


\section{Referencias Bibliográficas}

1. Santander XA., Rivera MC., Tipismana MA., Frecuencia de las señales de alarma en pacientes con cefalea, atendidos en la emergencia de un hospital de Lima.. Revista de Neuro-Psiquiatría [Internet]. 2013;76(2):101-108. Disponible de: http://portal.amelica. org/ameli/jatsRepo/372036944007

2.- Toledo JB, Riverol M, Martínez-Vila E, Irimia P. Cefalea en urgencias. Anales Sis San Navarra. 2008;31(1):75-85.

3.- Ardila GP. Cefalea en urgencias: diagnóstico y tratamiento de las cefaleas primarias. Acta Neurol Colomb. 2008;24(4):134-9.
4.- Locker TE, Thompson C, Rylance J, Mason SM. The utility of clinical features in patients presenting with nontraumatic headache: an investigation of adult patients attending an emergency department. Headache 2006; 46: 954-961.

5.- Valle J, Fonseca F, Vaquero M, De la fuente J, Llamas J, Hernandez Y. Cefalea súbita, punción lumbar y diagnóstico de hemorragia subaracnoidea en pacientes con una tomografía computarizada normal. Revista de la Sociedad Española de Medicina de Urgencias y Emergencias [Internet]. 2018 [citado 7 noviembre 2019];(Vol.30):50-53. Disponible en: https://dialnet.unirioja.es/ servlet/articulo? codigo $=6241345$
Correspondencia:

Mario Vera-Sanchez

marioverasanchez2015@gmail.com 